\title{
Bilateral testicular adrenal rests in a boy with congenital adrenal hyperplasia
}

\author{
Hee Kyung Kim • Eric Crotty
}

Received: 23 July 2010 /Revised: 19 August 2010 /Accepted: 10 September 2010/Published online: 7 October 2010

(C) Springer-Verlag 2010

A 16-year-old boy with a right testicular mass underwent scrotal US, which showed testicular masses in each testicle (arrow) with ill-defined hypoechoic spherical areas. In the left testicle, the tumor was located around the mediastinum (small arrows) (Fig. 1). The masses had slightly increased vascularity compared to the adjacent normal parenchyma. The patient had poorly controlled congenital adrenal hyperplasia (CAH) diagnosed neonatally. Given that history, US findings were compatible with bilateral testicular adrenal rests.

The adrenals and testes originate from the neighboring area in the embryo and the high level of ACTH in patients with $\mathrm{CAH}$ causes proliferation of aberrant adrenal cells in the testes, gonal dysfunction and infertility. The incidence of testicular adrenal rests in CAH varies and increases with age. In childhood, the prevalence is about $25 \%$ and mostly not palpable on physical exam [1]. A hypoechoic testicular mass without architecture distortion in patients with $\mathrm{CAH}$ is characteristic of adrenal rests, particularly as it is bilateral and should not be misinterpreted as bilateral testicular tumors such as lymphomas [2]. The mass is treated with steroids and rarely requires surgical enucleation when the adrenal rests do not respond to medical treatment $[1,2]$.
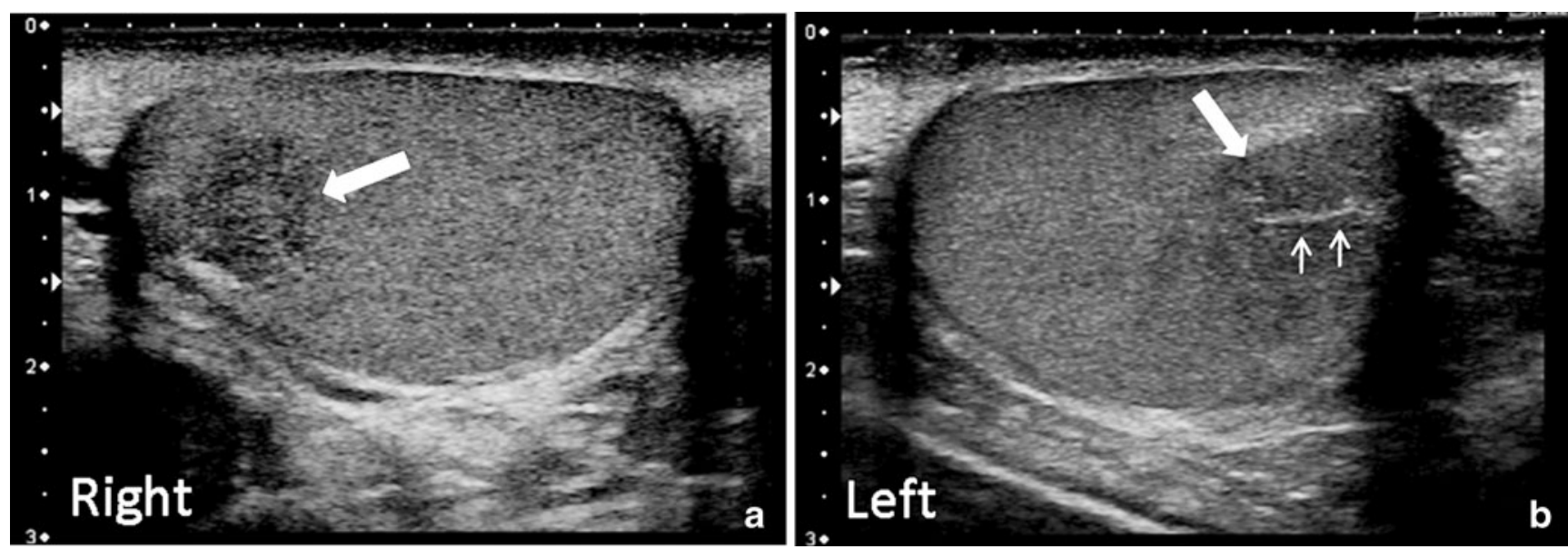

Fig. 1 Longitudinal US

\section{References}

\footnotetext{
H. K. $\operatorname{Kim}(\bowtie) \cdot$ E. Crotty

Department of Radiology,

Cincinnati Children's Hospital Medical Center,

3333 Burnet Ave.,

Cincinnati, OH 45229, USA

e-mail: Hee.Kim@cchmc.org
}
1. Claahsen-van der Grinten HL, Sweep FC, Blickman JG et al (2007) Prevalence of testicular adrenal rest tumours in male children with congenital adrenal hyperplasia due to 21-hydroxylase deficiency. Eur J Endocrinol 157:339-344
2. Vanzulli A, DelMaschio A, Paesano P et al (1992) Testicular masses in association with adrenogenital syndrome: US findings. Radiology 183:425-429

